\title{
Host transcription in active and latent tuberculosis
}

Sarah M Fortune and Eric J Rubin*

\begin{abstract}
A recent study has identified a transcriptional signature for active tuberculosis, suggesting that the distinction between active and latent forms may not be absolute.
\end{abstract}

Our understanding of tuberculosis (TB) is heavily colored by the available diagnostic tools. Active TB is identifiable first because of the presence of clinical symptoms, such as chronic cough, fever and weight loss. Active TB may be confirmed by the identification of the causative bacteria, Mycobacterium tuberculosis (Mtb), but in many cases bacteria are undetectable. Latent TB is defined by the presence of a T-cell-mediated immune response to Mtb in an asymptomatic individual. This immunological response to Mtb is widely believed to indicate the presence of viable organisms that might cause active disease in the future, although how many people with a detectable immune response actually harbor $\mathrm{Mtb}$ is unknown. The tests used to identify active and latent Mtb infection are among the oldest in modern medicine, dating back to the late 19th century. Thus, much of our understanding of human TB has been frozen for more than a century.

Can a systematic, molecular approach to understanding the host response to Mtb provide new insights into the course of human disease? A recent study by Anne O'Garra and her colleagues suggests that it can [1]. To characterize host responses to Mtb in an unbiased manner they chose to use whole-genome transcriptional profiling. They collected blood from HIV-negative patients from the UK with well-demonstrated active TB, those with positive tests for latent TB but no evidence of active disease and healthy controls. The investigators prepared RNA from these blood samples and hybridized it to microarrays containing more than 48,000 probes for human sequences. Focusing on a training set of samples, they used statistical tests and clustering to identify a set of 393 genes whose expression is significantly different between patients with active disease and those with latent or no disease.

*Correspondence: erubin@hsph.harvard.edu

Harvard School of Public Health, 665 Huntington Ave, Boston, MA 02115, USA
They then looked at the independent test and validation groups from the UK and South Africa, respectively, and found that the expression levels of these genes could discriminate powerfully between patients with active disease and those with no disease [1]. This discrimination was not perfect, however. For example, some patients with latent disease clustered with those with active TB. Conversely, some with clinically diagnosed disease lacked transcriptional markers. However, when patients were evaluated clinically, those with the most severe disease, at least as determined by the extent of abnormality on chest $\mathrm{X}$-ray, had much more pronounced transcriptional changes than those with minimal disease. In fact, the extent of disease correlated fairly well with the transcriptional anomalies, leading the authors [1] to propose that differences in profiles define a 'molecular distance to health. This metric improved when the disease was treated.

Of course, there are two sides to diagnostic criteria; a test might sensitively detect disease but might suffer from lack of specificity. Measuring a patient's temperature, for example, sensitively detects infection but does not discriminate among different causes. To test for specificity, the authors [1] compared the transcriptional profiles in ТВ patients with those seen in patients infected with group A Streptococcus or Staphylococcus aureus, which are Gram positive pathogens like Mtb, although they cause very different clinical syndromes, or afflicted with the autoimmune diseases systemic lupus erythematosus (SLE) or Still's disease, which, like Mtb, cause a chronic inflammatory state. Through these comparisons, the authors [1] were able to cull the list of transcripts to a set of 86 that were indicative of active TB.

Transcriptional profiles reflect both the composition of the sampled cell population and any changes in RNA abundance within those cells. O'Garra and colleagues [1] found that differences in circulating cells and changes in gene expression both contributed to the transcriptional signature that identified TB patients. For example, patients with active disease had a reduction in $\mathrm{T}$ cell transcripts that was associated with fewer circulating $\mathrm{CD}^{+}$and $\mathrm{CD}^{+} \mathrm{T}$ cells rather than a change in RNA abundance in these $\mathrm{T}$ cells, which was similar in patients and controls. On the other hand, whereas myeloid cell numbers were similar among the patient and control groups, their transcript content differed markedly. 
Interestingly, many of the differences seen between patients with active disease and those with latent or no infection were due to transcripts that are characteristic of cells, particularly neutrophils, produced in response to both interferon (IFN)- $\gamma$ and IFN- $\alpha \beta$. The identification of an IFN- $\alpha \beta$ response signature in a systemic bacterial infection is perhaps surprising for those who consider these cytokines characteristic of responses to viral pathogens. However, bacteria such as Mtb that reside within host cells and deliver bacterial material into the host cell cytosol activate cytosolic innate immune receptors and trigger an IFN- $\alpha \beta$ response [2]. Because group A Streptococcus and S. aureus do not similarly activate cytosolic pattern receptors, further work will be required to determine whether the 86-gene signature that O'Garra and colleagues [1] identified will distinguish Mtb from these kinds of bacteria, which include common pathogens such as Salmonella typhi, in addition to relevant viral infections such as HIV.

What do these transcriptional responses mean for the pathogenesis of disease? Although, like any observational study, the findings are only correlative, some of the factors identified [1] are well known to be important determinants of susceptibility to infection. In particular, individuals with abnormalities in IFN- $\gamma$ signaling pathways are far more likely to develop progressive disease after exposure to mycobacteria [3]. However, extrapolating to other genes might be difficult. In fact, by definition, patients with active TB are precisely those that failed to control initial infection. Thus, the transcriptional responses in these patients may mark a failure of immune control or reflect inflammatory responses to disease rather than TB-specific immune mechanisms.

Given this problem, it is interesting that the transcriptional signature [1] does not distinguish perfectly between active disease and latent infection. Between 10 and $25 \%$ of the latently infected individuals had transcriptional profiles that clustered with those from patients with active disease. It is intriguing to speculate that the overlap between active and latent disease is more than simply a statistical fluke. The transcriptional signatures indicative of active disease were more pronounced in individuals with greater radiographic evidence of disease, and they changed following treatment. These data suggest that the transcriptional signature reflects bacterial burden. Thus, it is possible that the patients with clinically latent disease but transcriptional signatures consistent with active disease have greater bacterial burdens than other latently infected patients.

In fact, recent evidence suggests that active and latent infections are not binary but, instead, represent different ends of a continuum of disease [4]. This new paradigm of TB is largely based on studies in nonhuman primates, including the cynomolgus macaque [5]. In these primates, active and latent disease seem to be overlapping entities rather than clearly distinct syndromes. The ability to measure Mtb burden in humans could help us toward a more relevant model of the diversity of disease states. Importantly, those with high burdens of organisms, as detected by transcriptional patterns, despite clinical latency might have the highest risk of progression to active TB.

Does the transcriptional signature identified by O'Garra and colleagues [1] provide a better way to diagnose active TB? Perhaps in the future; however, in many areas of the world where TB is now prevalent, even simple clinical ways to diagnose $\mathrm{TB}$, such as microscopic observation of sputum smears, taxes the available resources, and measuring hundreds of transcriptional parameters, or even a single transcript, is currently impossible. However, there are many findings from this study [1] that could be applied in research environments today. If transcriptional analysis truly can be used to measure the bacterial burden, it could be used to help understand the pathogenesis of disease and to predict those at highest risk of active disease. In addition, we currently have almost no way of measuring responses to treatment aside from assessing clinical parameters and relapse rates, both of which are slow and insensitive. A set of transcripts that correlates highly with treatment response could revolutionize how drug testing is performed.

The host response might well be an imperfect reflection of the molecular events that occur during TB. It is easy to believe that different individuals might vary in their response to a similar number of pathogens. In particular, those with HIV are likely to produce a very different transcriptional pattern from those with no immune compromise, and it will be critical to test the predictions of the current study in an HIV-infected population. Nevertheless, studies such as this one [1] represent a real opportunity to develop new insights into TB.

Published: 28 September 2010

References

1. Berry MP, Graham CM, McNab FW, Xu Z, Bloch SA, Oni T, Wilkinson KA, Banchereau R, Skinner J, Wilkinson RJ, Quinn C, Blankenship D, Dhawan R, Cush JJ, Mejias A, Ramilo O, Kon OM, Pascual V, Banchereau J, Chaussabel D, O'Garra A: An interferon-inducible neutrophil-driven blood transcriptional signature in human tuberculosis. Nature 2010, 466:973-977.

2. Leber JH, Crimmins GT, Raghavan S, Meyer-Morse NP, Cox JS, Portnoy DA: Distinct TLR- and NLR-mediated transcriptional responses to an intracellular pathogen. PLoS Pathog 2008, 4:e6.

3. Casanova JL, Abel L: Genetic dissection of immunity to mycobacteria: the human model. Annu Rev Immuno/ 2002, 20:581-620.

4. Barry CE 3rd, Boshoff HI, Dartois V, Dick T, Ehrt S, Flynn J, Schnappinger D, Wilkinson RJ, Young D: The spectrum of latent tuberculosis: rethinking the biology and intervention strategies. Nat Rev Microbio/ 2009, 7:845-855.

5. Lin PL, Pawar S, Myers A, Pegu A, Fuhrman C, Reinhart TA, Capuano SV, Klein E, Flynn JL: Early events in Mycobacterium tuberculosis infection in cynomolgus macaques. Infect Immun 2006, 74:3790-3803.

doi:10.1186/gb-2010-11-9-135

Cite this article as: Fortune SM, Rubin EJ: Host transcription in active and latent tuberculosis. Genome Biology 2010, 11:135. 